\title{
Televisiones que emiten en España sin licencia
}

\section{Television broadcasting in Spain without license}

\begin{abstract}
Álvaro Alás Brun: Asociación Plaza del Castillo de Usuarios de Medios de Comunicación aa.brun@gmail.com
\end{abstract}

\section{CURRÍCULUM VITAE}

Es Licenciado en Comunicación Audiovisual y miembro de la Asociación Plaza del Castillo de Navarra (España) sobre ética en la televisión.

\section{RESUMEN}

Desde la Asociación Plaza del Castillo de Usuarios de Medios de Comunicación, presentamos el informe que, como su propio título indica, trata de acercarse al controvertido asunto de las cadenas de televisión que han emitido y aún vienen emitiendo sin licencia en España. En el caso que nos ocupa, tratamos de analizar aspectos fundamentales de nuestra realidad audiovisual incomprensiblemente alejados de la preocupación de responsables políticos y mediáticos.

\section{PALABRAS CLAVE}

Usuarios - Informe - Cadenas de televisión - Licencia 


\begin{abstract}
From the Association of Users of Plaza del Castillo Media, introducing the report, as its title suggests, tries to approach the controversial issue of TV stations that have yet been issued and delivered without a license in Spain. In this case, try to analyze key aspects of our reality audiovisual inexplicably removed from the concern of policymakers and the media.
\end{abstract}

\title{
KEY WORDS
}

Users - Report - TV stations - Licence

\section{ÍNDICE}

1. Presentación por Maribel Martínez Éder

2. Introducción

3. Informe Jurídico, por Zuza Abogados

3.1 Infracciones en materia audiovisual y de telecomunicaciones

3.2 La regulación de las infracciones y sus formas

3.3 Anteproyecto de la Ley General Audiovisual

\subsection{Conclusión}

4. Pornografía en abierto: Incumplimiento de la normativa europea

4.1 Normativas europeas referidas a los contenidos audiovisuales

4.1.1 Convenio Europeo sobre televisión Transfronteriza hecho en Estrasburgo el 5 de Mayo de 1989

4.1.2 Directiva 2007/65/CE del Parlamento Europeo y del Consejo del 11 de Diciembre de 2007. Artículo 22

4.2 Ley 25/94, de 12 de julio. Ley vigente en España relativa al ejercicio de actividades de radiodifusión televisiva 
4.3 Informe jurídico por Zuza Abogados: Aplicabilidad de las directivas no transpuestas a la normativa estatal

4.3.1 Introducción al régimen de las directivas europeas

4.3.2 Aplicación directa de las directivas

4.3.3 Aplicación en materia televisiva

4.3.4 Interpretación de la normativa estatal, el efecto indirecto

5. Panorama de la televisión sin licencia en España

5.1 Consejo Audiovisual de Andalucía

5.1.1 Actuaciones ante la proliferación de televisiones sin licencia

5.1.2 El Presidente del CAA aboga por una futura Ley Audiovisual de Andalucía

5.1.3 Informe del CAA de la ponencia sobre la emisión de anuncios de contactos sexuales en televisión

5.2 Consejo Audiovisual de Cataluña

5.2.1 El CAC estudia sancionar a dos televisiones locales por emitir concursos y 'chats' de forma ilegal

5.3 Consejo Audiovisual de Navarra (Informes anuales del CoAN)

5.3.1 Informe 2004

5.3.2 Informe 2005

5.3.3 Informe 2006

5.3.4 Informe 2007

5.4 Consejo Audiovisual de la Comunidad de Madrid

5.5 Comunidad Autónoma de Aragón

5.6 Comunidad Autónoma de Murcia

5.7 Comunidad Autonómica Valenciana

6. Enmienda 177 que el Gobierno Socialista quiso plantear ante el Parlamento pero que finalmente retiró

6.1 Costes Generales. Diario de sesiones del Congreso de los Diputados

7. Conclusión 


\section{TEXTO}

\section{Presentación por Maribel Martínez Éder}

(Presidenta de la Asociación Plaza del Castillo de Usuarios y Medios de Comunicación de Navarra)

Desde la Asociación Plaza del Castillo de Usuarios de Medios de Comunicación, presentamos el informe que, como su propio título indica, trata de acercarse al controvertido asunto de las cadenas de televisión que han emitido y aún vienen emitiendo sin licencia en España. En el caso que nos ocupa, tratamos de analizar aspectos fundamentales de nuestra realidad audiovisual incomprensiblemente alejados de la preocupación de responsables políticos y mediáticos.

El estudio ha sido realizado por el miembro de la asociación Álvaro Alás Brun, licenciado en Comunicación Audiovisual, y se encuadra en Cuadernos para la Información y Formación de los Usuarios de Medios de Comunicación, en colaboración con la sección de Consumo del Gobierno de Navarra.

Es preciso recalcar que no ha sido nada fácil obtener información al respecto, puesto que no se disponen, que sepamos, datos oficiales, y nuestras únicas fuentes de información con las deficiencias que explicaremos, han sido, entre otros, los tres consejos audiovisuales que existen en España, por orden alfabético: el de Andalucía, el de Cataluña y el de Navarra. Extrapolando los datos que hemos obtenido y aludiendo a informaciones fiables, analizamos un asunto muy delicado y que como queda claro, no hay organismo público ni privado que a lo largo de los últimos años haya conseguido encarar.

El fin del trabajo realizado ha sido el de aproximarnos, estudiar y divulgar una situación irregular de la que venimos preocupándonos en pleno apagón analógico, y 
la correspondiente transformación en tecnología digital que cambiará a partir de hoy, por fuerza, el panorama drásticamente. Queremos resaltar la importancia del momento porque el vuelco será definitivo, aunque no elimina la responsabilidad de los poderes públicos durante todos los años que permitieron una situación de irregularidad en la transposición de la normativa europea, y de fraude en relación con los contenidos y la responsabilidad de las empresas.

Contenidos sin ningún control

Hay que partir de la base de que las cadenas sin licencia emiten contenidos sin ningún control, generalmente fraudulentos: adivinadores, tarots, echadoras de cartas, así como chats y pornografía dura en abierto y frecuentemente en horarios de protección. Utiliza, además, con el consiguiente perjuicio hacia las personas más desfavorecidas cultural y económicamente, teléfonos de tarifación adicional, con la particularidad de que las condiciones son ilegibles.

La normativa europea (1989), de obligado cumplimiento en todos los países miembros, especifica claramente que no se puede emitir pornografía en abierto. La ley española 25/94 prohíbe emitir lo que perjudica seriamente el desarrollo físico, mental o moral, pero no especifica qué es "perjudicar seriamente", expresión suficientemente ambigua que ha hecho posible la situación que hemos venido padeciendo.

Pamplona- Madrid. Junio de 2009 


\section{Introducción}

La nueva ley de lo Audiovisual que el gobierno de D. José Luís Rodríguez Zapatero ha prometido elaborar antes de que se acabe su mandato tiene entre sus prioridades la de multar y cerrar aquellas televisiones que operen sin licencia.

Mientras tanto, en las tres comunidades autonómicas que tienen un consejo audiovisual (Cataluña, Andalucía y Navarra), cuando llega alguna denuncia sobre contenidos ilícitos de cadenas que emiten sin licencia, lo derivan al Ministerio de Industria, Comercio y Turismo del Gobierno Central. Éste les responde diciendo que pueden sancionar, pero sólo a aquellas que emitan desde su territorio. Como las emisiones de la mayoría (como Telelínea) llegan desde otra autonomía, los Consejos Audiovisuales no les pueden multar, llegando la denuncia al Ministerio de Industria, Comercio y Turismo, que las archiva en espera de la nueva Ley de lo? Audiovisual.

De este modo se están beneficiando televisiones ilegales, que emiten en abierto contenidos fraudulentos, canales de tarot, anuncios de relaciones sexuales, pornografía, etc., a horas incluso de espacio protegido a menores de edad. Todo ello en detrimento de los espectadores que se encuentran desprotegidos, como en ningún otro país de Europa.

El estudio refleja la cantidad de televisiones que emiten sin licencia en España, las denuncias que ha habido contra ellas y quiénes son los responsables de que sigan existiendo. 


\section{Informe Jurídico, por Zuza Abogados}

\subsection{Infracciones en materia audiovisual y de telecomunicaciones}

El art. $149.1 .21^{\circ}$ de la Constitución, establece que el régimen general de telecomunicaciones es competencia exclusiva del estado, y a su vez, el apartado $1.27^{\circ}$ de dicho artículo, prescribe la competencia exclusiva del estado en materia de comunicación social, exponiendo de forma expresa, los medios de prensa, radio y televisión, lo que incluye la disciplina objeto de estudio.

El mismo precepto, (art. 149.1.27 ), propone en una redacción un tanto contradictoria, la posibilidad de desarrollo de la legislación estatal por las CCAA, desarrollo de una materia cuyas facultades están atribuidas de forma exclusiva al Estado, expresando además de la facultad de desarrollo la de ejecución. A pesar de la redacción poco afortunada del precepto, encontramos su fundamentación en el art. 150.1, que propone el desarrollo por las Comunidades Autónomas, de las competencias estatales.

En cuanto a la normativa reguladora, consideraremos la siguiente legislación:

Ley 32/2003 de 3 de noviembre, General de Telecomunicaciones

Ley 25/1994 de 12 de junio que traspone la directiva 89/552/CEE

Ley 22/1999, que viene a modificar a su vez a la Ley 25/1994

Ley Foral 18/2001 de 5 de julio

Anteproyecto de Ley General Audiovisual 


\subsection{La regulación de las infracciones y sus formas}

En la actualidad, a falta de una regulación que refunde la normativa vigente, bastante dispersa y parca, serán de aplicación en la disciplina, los arts. 50 y ss de la Ley General de Telecomunicaciones, en la materia prescrita en el art. 1.1 de la misma norma, que se refiere al art. $149.1 .21^{\circ} \mathrm{CE}$, pero no al $149.1 .27^{\circ}$, por lo tanto, habremos de distinguir que el ap. $21^{\circ}$ se refiere, a una cuestión de medios de comunicación destinados a un servicio de convivencia sin dilación a la hora de entablar los negocios de la vida, pensando en las telecomunicaciones más como medios de transporte, que como medios de transmisión de información; sin embargo, el apartado $27^{\circ}$ regula concretamente los medios.

Entrando en materia, cabe distinguir infracciones muy graves, graves y leves, sobre la base del art. 52 y de los arts. 53 y ss. de la Ley General de Telecomunicaciones.

En cuanto a las sanciones, los órganos competentes, según el ámbito de sus respectivas competencias, son, el Ministerio de Ciencia y Tecnología, y la Comisión del Mercado de las Telecomunicaciones, imponiendo multas y medidas cautelares y de cesación, según el tipo de infracción, art. 56 de la Ley General de Telecomunicaciones, sobre la base de la Ley 30/1992 reguladora de las Administraciones Públicas, puesto que se trata de dos órganos administrativos.

Como se puede apreciar, esta norma, regula los supuestos de infracción formal, en materia de instalaciones, pero no contempla los supuestos de emisión de programación concreta, especialmente en los supuestos de emisión de contenido perjudicial para los menores. Para ello, habremos de estar a la Ley 25/1994 de 12 de julio y a su análoga en Navarra, la Ley 18/2001 de 5 de julio, (sin perjuicio de lo dispuesto en otras disposiciones autonómicas). 
Esta Ley establece un sistema sancionador propio de las CCAA dentro de su ámbito territorial y para el resto de supuestos, el poder central, art. 19.

La potestad sancionadora del Estado, la ejerce el Ministerio de Fomento, mientras que las CCAA establecerán el órgano administrativo que consideren oportuno.

Las infracciones son calificadas de graves o muy graves, (art. 20) y el régimen a aplicar es el previsto en la Ley 30/92 reguladora de las Administraciones Públicas.

De ahí, que sin entrar a cada tipo de infracción, se establecen en estas dos leyes, dos regímenes, uno que regula la disciplina desde un punto de vista estructural, es decir, todo aquello relacionado con la instalación, comunicación a la Administración, concesión de licencias, ocupación del espacio público etc., y otro que regula el contenido de la emisión, si es lícito o no, según el horario y el espectador al que va dirigido, sin perjuicio de aquellos contenidos radicalmente prohibidos, que no gozan de horario de cortesía.

\subsection{Anteproyecto de la Ley General Audiovisual}

En cuanto al anteproyecto de Ley General Audiovisual, este establece una nueva regulación de la TDT, (Televisión Digital Terrestre) y va encaminada a la desaparición del sistema analógico de telecomunicaciones, (es decir, el que mediante una antena sobre el tejado redirigimos la señal mediante un cable hasta nuestro televisor) con la nueva entrada del sistema digital, que funciona a través de fibra óptica o sucedáneo.

Otra de las propuestas es la liberalización del sector audiovisual dejando de ser servicio público esencial, que a mi juicio vulnera el art. 20.1 d) de la Constitución. 
Pueden seguir existiendo televisiones públicas, pero se limitan a un canal analógico y cuatro digitales, en total cinco canales, el resto es televisión privada salvo un canal que se permite a las televisiones locales.

De modo más formal que material, se promueve el pluralismo lingüístico y se exigirá a pesar de la liberalización, que las televisiones locales emitan programas concretos y medidas de transparencia, como el conocimiento por parte de los ciudadanos de los propietarios de los medios de comunicación y la inclusión de una acción de cesación por parte de los ciudadanos para poner fin a la emisión de contenido ilícito, inmoral, fuera de su horario de cortesía o radicalmente opuesto a la ley.

\subsection{Conclusión}

A mi juicio, la legislación actual en esta materia tiene un régimen completo, que establece un cuadro de infracciones y sanciones muy amplio, cierto es que con una gran complejidad, pero entiendo que en esta materia no es tan necesaria una modificación de la normativa en vigor, cuanto una mayor voluntad política de la Administración competente de ejercer sin paliativos el poder sancionador que detenta, pero que apenas aplica.

\section{Pornografía en abierto: Incumplimiento de la normativa europea}

La normativa europea especifica que no se puede emitir pornografía en abierto. Nuestra ley 25/94 prohíbe emitir lo que perjudica seriamente pero no nos especifica lo que es "perjudicar seriamente", con lo que prácticamente no podemos denunciar a ninguna emisora que ponga contenidos pornográficos. 


\subsection{Normativas europeas referidas a los contenidos audiovisuales}

4.1.1 Convenio Europeo sobre televisión Transfronteriza hecho en Estrasburgo el 5 de Mayo de 1989

\section{Capítulo II}

Disposiciones relativas a la programación

Art. 7. $^{\circ}$ Responsabilidades del radiodifusor. -1 . Todos los elementos de los servicios de programas, por su presentación y contenido, deberán respetar la dignidad de la persona humana y los derechos fundamentales ajenos.

En particular, no deberán:

a) Ser contrarios a las buenas costumbres y en especial contener pornografía.

b) Prestar relieve a la violencia ni incitar al odio racial.

\subsubsection{Directiva 2007/65/CE del Parlamento Europeo y del Consejo del 11} de Diciembre de 2007. Artículo 22

Artículo 22 Protección de los menores

1. Los Estados miembros adoptarán las medidas oportunas para garantizar que las emisiones de televisión de los organismos de radiodifusión televisiva bajo su jurisdicción no incluyan ningún programa que pueda perjudicar seriamente el desarrollo físico, mental o moral de los menores y, en particular, programas que incluyan escenas de pornografía o violencia gratuita.

4.2 Ley 25/94, de 12 de julio. Ley vigente en España relativa al ejercicio de actividades de radiodifusión televisiva 
Artículo 17.- Protección de los menores frente a la programación

1. Las emisiones de televisión no incluirán programas ni escenas o mensajes de cualquier tipo que puedan perjudicar seriamente el desarrollo físico, mental o moral de los menores, ni programas que fomenten el odio, el desprecio o la discriminación por motivos de nacimiento, raza, sexo, religión, nacionalidad, opinión o cualquier otra circunstancia personal o social.

\subsection{Informe jurídico por Zuza Abogados: Aplicabilidad de las directivas no transpuestas a la normativa estatal}

\subsubsection{Introducción al régimen de las directivas europeas}

La Directiva es una disposición normativa, normalmente sucinta, que contempla un mandato a los estados miembros, de promulgar una norma a través de su régimen interno que contemple una regulación de los aspectos mencionados en la Directiva y al tenor de la misma, es decir, bajo sus directrices. En algunos supuestos, la Directiva puede ser directamente aplicable e incluso los ciudadanos excepcionalmente pueden instar su aplicación ante los tribunales, cuando la misma sea lo suficientemente clara y concisa y el plazo de transposición otorgado por la CE no haya transcurrido.

\subsubsection{Aplicación directa de las directivas}

Existen dos tipos de aplicación directa, el efecto directo vertical, que consiste en la aplicación directa entre ciudadanos y la Administración del Estado, (normalmente limitada a la solicitud de daños y perjuicios al Estado por no haber llevado a cabo la transposición en plazo), aceptada por la Jurisprudencia del Tribunal de Justicia de las Comunidades Europeas, y el efecto directo horizontal, esto es, entre particulares, cuya aplicación material no esta permitida, aunque sea posible su invocabilidad. El 
problema radica en que el estado no puede exigir las eventuales obligaciones al ciudadano.

\subsubsection{Aplicación en materia televisiva}

Bajo un análisis sucinto del supuesto, podemos afirmar que no procede la aplicabilidad material de la Directiva 2007/65/CE. ¿Cómo es posible? La razón estriba en que la aplicabilidad directa solo procede entre la Administración del Estado y los ciudadanos, es decir, frente al poder ejecutivo y no frente a aquellos órganos mediáticos que el estado pueda regentar que no poseen capacidad ablativa sobre los ciudadanos.

\subsubsection{Interpretación de la normativa estatal, el efecto indirecto}

La exégesis de nuestras normas, frente a las disposiciones europeas, debe llevarse a cabo conforme a estas últimas, Si bien no cabe la invocación directa de las Directivas salvo las excepciones plasmadas.

Esto podemos articularlo de modo que ha de considerarse aplicable el artículo 22 de la directiva a través de la interpretación del Derecho supranacional. Citaremos la STJCE Von Colson y Kamann, en la que se establece la obligación de los tribunales estatales de interpretar la normativa interna a la luz de las directivas europeas, tesis concretada en la sentencia sobre el asunto Kolpinghuis Nijmegen, $n^{\circ}$ 80/86, de 8 de octubre de 1987, al afirmarse, en primer lugar, que, no obstante, dicho efecto indirecto no puede dar lugar a una interpretación contraria a las leyes estatales y, en segundo lugar, que el efecto indirecto será aplicable con independencia de que no haya prescrito todavía el plazo de transposición de la directiva. 
Un ulterior paso se dio, finalmente, con la sentencia sobre el asunto "Marleasing", $\mathrm{n}^{\circ}$ 106/89, de 13 de noviembre de 1990, que, sin admitir el efecto horizontal, determina que las lagunas legales del ordenamiento interno han de colmarse, en interpretación conforme con el Derecho comunitario, también en litigios entre particulares.

\section{Panorama de la televisión sin licencia en España}

\subsection{Consejo Audiovisual en Andalucía}

\subsubsection{Actuaciones ante la proliferación de televisiones sin licencia}

Según Carmen Fernández Morillo, Consejera del Consejo Audiovisual de Andalucía (CAA), la creación en Andalucía de una autoridad independiente y reguladora del sector audiovisual, como es el CAA, se produce en un momento de tránsito de la televisión analógica a la digital que en la comunidad autónoma se empieza a desarrollar en abril del 2006, cuando el Gobierno inicia los procedimientos para otorgar 258 licencias de ámbito local, 186 privadas y 72 a ayuntamientos.

Además de un salto tecnológico, la implantación de la TDT -con la fecha del 2010 como horizonte- conlleva un hecho importante: la Consejería de Presidencia de la Junta asume la ordenación de un sector, el de la televisión local, que se ha desarrollado de manera espectacular en una situación de precariedad normativa y sin control administrativo.

El Consejo Audiovisual asume en octubre del 2005 la responsabilidad de seguir los contenidos televisivos en un escenario conformado por un ingente pero indeterminado número de televisiones locales con tres estatus jurídicos distintos. El escenario es en realidad una selva mediática donde conviven televisiones que emitían antes del 1 de enero de 1995 y que funcionan aparándose en una habilitación transitoria de la Ley 41/1995, de 22 de diciembre, de Televisión Local por Ondas Terrestres. Aquellas creadas entre esa fecha y enero de 2004 y las que empezaron a 
funcionar tras esa fecha, sin cobertura legal alguna. Llanamente se les distinguía como legales, alegales e ilegales.

El Consejo no sólo no ha podido disponer de un censo fiable y real de televisiones locales sino que la mayoría operaban bajo el manto protector que genera la incertidumbre legislativa y la inseguridad jurídica.

La tardanza de la Administración estatal en planificar la reserva de frecuencias y en dictar el plan técnico correspondiente demoró la convocatoria de los concursos para el otorgamiento de la concesión, provocando esta situación.

En cualquier caso, existía ya una legislación audiovisual plenamente vigente pero hasta la creación del Consejo, en Andalucía no se estaba vigilando ni actuando para asegurar que se respetase.

Uno de los primeros interrogantes que debió resolver el Consejo fue precisamente si podía ejercer su potestad inspectora y sancionadora en el conjunto del sector, independientemente de su situación jurídica y administrativa, o -por el contrariolimitaba sus actuaciones al reducido número de operadores que disponían de título habilitante, tal como podría desprenderse de una somera lectura la Ley 1/2004.

Se optó por lo primero, aun conociendo las dificultades y complicaciones administrativas y jurídicas que conllevaría aplicar la legislación audiovisual sobre contenidos audiovisuales a emisoras clandestinas y sobre un sector que se había desarrollado sin vigilancia ni control alguno.

En el 2007, el Consejo puso en marcha un costoso y complejo sistema de seguimiento de contenidos que permite, de manera sistemática, ver y grabar cada día en torno a 600 horas de televisión emitidas por el operador público autonómico y emisoras locales con un ámbito de influencia de más de 100.000 habitantes, distribuidas por toda la geografía andaluza, así como aquellas emisiones específicas para Andalucía 
de operadores estatales. Cuando esté totalmente desarrollado, el sistema permitiría visionar y grabar diariamente 1.200 horas de televisión y 1.000 de radio. A ello se suma un segundo sistema de seguimiento, a través de la red creada para vigilar el espacio radioeléctrico por la Consejería de Presidencia con la que se firmó un convenio de uso y desarrollo. Hasta entonces, nadie había asumido en España un reto semejante en un escenario tan incierto.

El objetivo de este seguimiento es velar por el cumplimiento de la legislación audiovisual, especialmente en horario de protección infantil, y realizar estudios e informes que respalden las decisiones, recomendaciones e instrucciones del Consejo dirigidas al sector.

Este seguimiento diario a los contenidos emitidos en Andalucía ha generado en el 2007 una base de datos con alrededor de 30.000 piezas de vídeo - 17.000 provenientes de operadores locales y 13.000 de la RTVA- para su estudio por el Servicio de Análisis del Consejo.

Sin embargo, las previsiones de velar para que las televisiones ofreciesen en la franja horaria protegida contenidos adecuados a la infancia y la juventud o vigilar por el cumplimiento de normas importantes -como las referidas a la señalética o los tiempos de publicidad- se vio superada por un problema mayor: la emisión de pornografía y publicidad de servicios sexuales en abierto, a cualquier hora del día.

La lucha contra la emisión entre las 6 y las 22 horas de pornografía y anuncios o mensajes de contactos sexuales en la televisión local ha sido una prioridad para esta institución. Poner fin a esta práctica extendida en la televisión local ha absorbido la mayor parte de los recursos del Consejo como consecuencia, sobre todo, del incierto y complejo escenario ya descrito. En julio del 2007, se remitió masivamente un escrito recordando la legislación para que los operadores locales adecuasen sus contenidos a lo establecido por la Ley de Televisión sin Fronteras. Se advirtió a todas las 
televisiones locales ya fueran legales, alegales o clandestinas detectadas a través de los diferentes sistemas de seguimiento que, a partir de septiembre, el Consejo expedientaría a quienes infringiesen gravemente la normativa emitiendo pornografía o mensajes de contactos sexuales en franja protegida.

La apertura de los primeros expedientes sancionadores tropezó, sin embargo, con la dificultad de identificar y localizar a quienes emitían dichos contenidos, misión que en muchos casos ha resultado imposible por mucha voluntad y esfuerzo que se ha puesto en ello. El mapa audiovisual andaluz cambiaba cada día, con televisiones que entran y salen de una frecuencia, aparecen y desaparecen o no se sabe a ciencia cierta quiénes son sus responsables.

El Pleno del Consejo consideró prioritaria la actuación contra la pornografía televisiva y, en ese sentido, ordenó un rastreo que tenía como objetivo detectar a aquellos operadores que, en el conjunto del territorio andaluz, pudieran emitir imágenes y mensajes de contenido sexual explícito en horario protegido.

El 19 de marzo de 2008 se realizó este rastreo general, entre las 12 y las 21 horas, que arrojó un alarmante resultado: Del total de 397 canales sintonizados, 48 emitían pornografía y/o anuncios de mensajes explícitos de servicios sexuales. El grueso de la programación de otro medio centenar no era ilícita pero sí preocupante ya que consistía en programas de videncia, teletiendas y concursos con sms o tarificación adicional no dirigidos a menores de edad.

Ya en enero del 2008, el Consejo había trasladado a la Fiscalía Superior de Andalucía, al Gobierno y al Parlamento autonómico un informe sobre publicidad de contactos sexuales y prostitución en la televisión local, en el que mostraba su preocupación porque la ausencia de regulación de estos anuncios, y también de la pornografía, permitiese su libre emisión en abierto entre las 22 y las 6 horas. 
El Consejo considera en su informe que no se puede obviar la realidad social y que, para asegurar una efectiva protección de los menores, Andalucía puede regular y limitar aún más la emisión de contenidos pornográficos y el comercio sexual a través de la televisión, equiparándonos a otros países de Europa que además de adecuar su franja protegida a los hábitos horarios de la población prohíben la emisión en abierto de contenidos pornográficos.

Por otra parte, tras el rastreo de pornografía realizado en marzo, el Consejo decidió también intervenir requiriendo a los infractores el cese inmediato de dichos contenidos y proceder al mismo tiempo a la apertura de expedientes informativos, previos a los sancionadores. Además se ha implicando a otras instituciones, como la Fiscalía y la Dirección General de Comunicación Social de la Junta para que, desde sus respectivos ámbitos de competencia, lleven a cabo las actuaciones pertinentes.

Así, el Consejo está informando tanto a la Fiscalía como a la Dirección General de Comunicación Social de la Junta, responsable de las licencias, de todas las incidencias en relación con aquellas televisiones que siguen emitiendo pornografía o mensajes de contactos sexuales en horario protegido. En ese sentido, hay que resaltar que la mayoría de los operadores han atendido puntualmente los requerimientos en relación con la pornografía y la publicidad de servicios sexuales, si bien está resultando más complicado acabar con los mensajes de contactos sexuales insertos en chats. En este caso, los operadores argumentan problemas técnicos para que sus sistemas de control discriminen determinadas palabras no explícitas.

En julio de 2008, la Fiscalía Superior de Andalucía ha respaldado la estrategia del Consejo en su diligencia informativa 6/2008 donde considera particularmente necesario instar al respeto a los horarios de protección de menores y plantea que la desobediencia a los requerimientos de cese realizados por la institución pueda tener consecuencias penales para el operador de televisión. 
La previsión es que, en Andalucía, desaparezcan en el plazo de unos meses los operadores que emiten ilegalmente y que, bien por intervención de la Fiscalía o de la Dirección General de Comunicación Social, sean cerrados de manera inmediata aquellos que emiten contenidos pornográficos.

El CAA ha abierto unos veinte expedientes por emisión de pornografía y anuncios de contactos sexuales en franja infantil.

\subsubsection{El Presidente del CAA aboga por una futura Ley Audiovisual de} Andalucía (18 de diciembre de 2007)

El Presidente del Consejo Audiovisual de Andalucía (CAA), Manuel Ángel Vázquez Medel, ha comparecido hoy, jueves, ante el Parlamento de Andalucía, en la Comisión de Coordinación, para presentar el informe preceptivo que el CAA debe elevar a la Cámara cada año.

En su intervención, el Presidente del CAA después de hacer balance de las actuaciones llevadas a cabo durante el 2006, ha situado entre uno de sus objetivos futuros en "terminar" con los operadores ilegales o alegales antes de la implantación en Andalucía de la TDT (televisión digital terrestre) para garantizar una regulación "adecuada".

Por otra parte, se ha mostrado partidario de que el Parlamento de Andalucía ponga en marcha la próxima legislatura un proyecto de ley general audiovisual para Andalucía, con la pretensión de "armonizar" el marco normativo y ofrecer garantías jurídicas tanto a los operadores como al ejercicio de la autoridad del CAA. 


\subsubsection{Informe del CAA de la ponencia sobre la emisión de anuncios de} contactos sexuales en televisión

Es evidente que, con la actual normativa, los menores de edad tienen en España fácil y libre acceso a los contenidos contra los que previene la directiva comunitaria, situación que se ha agravado con el irregular desarrollo de la televisión local, que ha crecido espectacularmente en los últimos casos al margen, en muchos casos, de la legalidad o en condiciones de alegalidad. Esta falta de control ha permitido que televisiones de ámbito local desarrollen contenidos no convencionales, tanto en lo referente a la programación como en lo relativo a publicidad. Los anuncios de contactos sexuales constituyen un buen ejemplo de ello, aunque no el único.

\subsection{Consejo Audiovisual de Cataluña}

El Consejo Audiovisual de Cataluña (CAC) ha abierto expediente, a fecha de hoy, a 60 emisoras de televisión que operaban sin licencia, siguiendo un proceso parecido al de la noticia que se presenta a continuación.

5.2.1 El CAC estudia sancionar a dos televisiones locales por emitir concursos y 'chats' de forma ilegal

(BARCELONA, 3 de Noviembre de 2004) (EUROPA PRESS)

El Pleno del Consell de l'Audiovisual de Catalunya (CAC) acordó hoy abrir un periodo de información, previo al expediente sancionador, a la sociedad mercantil Infraestructuras y Gestión 2002 S.L., con sede en Vitoria, por los contenidos emitidos en Barcelona en los canales 28 y 35, por emitir publicidad presuntamente engañosa y 'chats' de contactos personales de carácter erótico en horario protegido. 
De este modo, la empresa denunciada dispone ahora de un plazo máximo de cinco días hábiles para formular las manifestaciones que considere oportunas, antes de que el CAC presente un expediente sancionador, dado que según el CAC, "la empresa no dispone de la autorización administrativa que prevé el Decreto 15/2003 por el que se regula el régimen jurídico transitorio de las televisiones locales".

El ente regulador ha recibido quejas procedentes mayoritariamente de Barcelona pero también de otras ciudades catalanas sobre los contenidos emitidos a través de los dos canales. En concreto, el CAC ha hecho el seguimiento de la señal recibida en Barcelona.

Los servicios del CAC identificaron el canal 28 a través del cual se emite una programación, identificada con el logotipo MX, basada principalmente en concursos. Los espectadores pueden participar a través del teléfono con prefijo 806 y mensajes SMS "sin que se incluyan todos los datos esenciales sobre estos servicios de tarifas adicionales", informó el CAC a través de un comunicado.

Asimismo, el canal 28 emite publicidad de servicios de videntes sin estar identificada como tal, con lo que podría constituir publicidad engañosa.

Por otra parte, el canal 35, identificado también como MX, emite a través de un formato de pantalla partida, 'chats' de contactos personales de carácter erótico, publicidad de servicios eróticos y publicidad para descargarse melodías en horario protegido, con lo que el CAC considera que "podría vulnerar la ley de atención y protección de los niños y adolescentes". Además, añade que "el uso que se hace de los sistemas de tarifas adicionales puede constituir publicidad engañosa".

En cuanto a la mecánica del concurso, en el que se formula una pregunta muy sencilla que ningún espectador acierta y muchas personas quedan retenidas al teléfono de tarifas adicionales, el CAC ha puesto en conocimiento de estos hechos al 
fiscal jefe del Tribunal Superior de Justicia de Catalunya, Josep Maria Mena, por si puede pueden ser considerados delitos.

El CAC también ha dado a conocer los hechos a la Secretaría de Estado de Telecomunicaciones y Sociedad de la Información, a la Comisión del Mercado de las Telecomunicaciones y a las operadoras Retevisión, Tradia-Difusión Digital Sociedad de Telecomunicaciones y Red de Banda Ancha de Andalucía.

\subsection{Consejo Audiovisual de Navarra (Informes anuales del CoAN)}

Según Borja Mora-Figueroa, miembro? del Consejo Audiovisual de Navarra (CoAN), esta institución, a lo largo de sus años de actividad, se ha enfrentado a la existencia de diversas televisiones que emiten sin autorización en el territorio de la Comunidad Foral de Navarra.

La inestabilidad del marco legal que debía regular esta situación propició la multiplicación de televisiones que emitían sin la pertinente autorización administrativa. Es preciso señalar, en todo caso, la gran variedad de modelos de televisiones que se fueron creando, algunas con programación de relativa calidad, otras con contenidos centrados en pornografía, videncia, chats o concursos telefónicos.

A pesar de que la Ley Foral 18/2001, de 5 de julio, que regula la actividad audiovisual en Navarra y crea el Consejo Audiovisual de Navarra, erige al CoAN como la "autoridad audiovisual en Navarra", el órgano competente para ejercer la labor de inspección, control y sanción de las televisiones que operan sin título habilitante es, en el ámbito geográfico de la Comunidad Foral de Navarra, el Gobierno de Navarra. Por esta razón, el CoAN ha trasladado las infracciones detectadas en este sentido al Gobierno de Navarra. 
En opinión del CoAN, la implantación definitiva de la Televisión Digital Terrestre (TDT) en España debe suponer el cese de las emisiones de las televisiones que no cuentan con la pertinente autorización administrativa. Las administraciones encargadas de vigilar el cumplimiento de esta situación deberán trabajar intensamente para que no se vuelva a repetir la proliferación de operadores que emitan sin licencia, especialmente cuando se produzcan los sucesivos "apagones analógicos" a lo largo de 2009 y de 2010.

\subsubsection{Informe 2004}

Telelínea TV

En agosto de 2004, el Consejo Audiovisual de Navarra detecta la existencia de una emisora de televisión que opera bajo el nombre comercial de Telelínea y que no cuenta con la correspondiente concesión administrativa. Este canal basa su programación en la difusión de contenidos tipo: videncia, consultorios sentimentales, vídeos musicales, chats y pornografía, en su mayoría. En los programas y anuncios se hace promoción de números de teléfono de tarificación adicional (806,803 y 807) animando a la participación del espectador. Dado que la emisora no dispone de licencia para la prestación del servicio de televisión y que sus contenidos podrían constituir un supuesto de publicidad engañosa así como una vulneración del horario de protección del menor, el Consejo Audiovisual de Navarra decidió, en sesión de 9 de septiembre de 2004, denunciar estos hechos a la Comisión del Mercado de las Telecomunicaciones (CMT) y a la Secretaría de Estado de Telecomunicaciones y para la Sociedad de la Información. El Consejo entendió que la competencia sancionadora era de estos organismos, puesto que las emisiones de la cadena se realizaban tanto por satélite como por ondas hertzianas, pero con un centro emisor situado fuera de Navarra. 
El 24 de diciembre de 2004, se recibe contestación de la Secretaría de Estado de Telecomunicaciones. En ella se indica que, en cuanto a los números de tarificación adicional, la denuncia se deriva a la Comisión de Supervisión del Código de Conducta para la prestación de los Servicios de Tarificación Adicional, por ser el organismo competente para verificar si Telelínea incumple dicho Código. En cuanto a la prestación del servicio de televisión, la Secretaría de Estado se compromete a estudiar el tema pero nos recuerda que la competencia para controlar y sancionar estos hechos, en el caso de que existieran remisores en Navarra o se tratara de una televisión local, pertenece al Gobierno de Navarra. Quedando pendiente de recibir las actuaciones realizadas por la CMT, este Consejo continuará con el procedimiento durante 2005 y adoptará las medidas que se estimen oportunas.

\subsubsection{Informe 2005}

Informe sobre la situación legal de determinadas televisiones que emiten en Navarra a raíz de la denuncia de un particular

El 26 de mayo de 2005 tuvieron entrada en el Consejo Audiovisual de Navarra cinco denuncias presentadas por un particular por la supuesta existencia de una serie de televisiones que emiten en la Comunidad Foral de Navarra sin la pertinente concesión administrativa. Las denuncias fueron remitidas a este órgano desde la Subdirección General de Ordenación de las Telecomunicaciones del Ministerio de Industria, Turismo y Comercio, pasando por la Consejería de Economía y Hacienda y por el Director General para la Sociedad de la Información del Gobierno de Navarra. A la vista de los escritos del demandante, el Consejo aprobó el 26 de julio de 2005 un informe que se realizó a iniciativa propia, tal y como permite el artículo 26. 1. a) de la Ley Foral 18/2001. En él, el Consejo entendía que la suspensión de las emisiones de las televisiones por el uso ilegal del espectro radioeléctrico era atribución propia del Ministerio de Industria, Turismo y Comercio por lo que, al ser éste el principal 
motivo de las denuncias presentadas, era su competencia realizar las acciones demandadas por el denunciante.

En lo referente al segundo punto de las denuncias, la emisión de señales televisivas sin la correspondiente autorización administrativa, la jurisdicción del Consejo Audiovisual de Navarra se circunscribía a la imposición de sanciones por emitir sin las pertinentes licencias, tal y como se contempla en los artículos 18 y 19 de la Ley Foral 18/2001. En este sentido, y como paso previo al inicio de los correspondientes expedientes sancionadores, el Consejo abrió un periodo de información previa para esclarecer los hechos y conocer las circunstancias concretas a la hora de proceder a la imposición de sanciones. Pero hubo de hacer constar que, dado el tiempo transcurrido desde que el reclamante presentó la primera denuncia ante el Ministerio, dos de las emisoras de televisión a que se refería la misma ya poseían autorización para emitir, por lo que no incurrían en incumplimiento alguno.

El informe fue trasladado al Subdirector General de Ordenación de las Telecomunicaciones del Ministerio de Industria, Turismo y Comercio, al Consejero de de Economía y Hacienda del Gobierno de Navarra y al Director General para la Sociedad de la Información.

\subsubsection{Informe 2006}

Expedientes sancionadores

Televisión

Durante el año 2006, continuó la tramitación, por parte del Instructor, del Expediente Sancionador 1/2005 abierto contra la empresa Telelínea Local S.A. 
Tras la presentación de las alegaciones por parte del interesado, se acordó la apertura de un periodo probatorio, a cuya conclusión el Instructor dictó, con fecha 15 de junio de 2006, una resolución en la que se acordó elevar el expediente al CoAN para su resolución o, en su caso, para la ordenación de la práctica de las diligencias complementarias.

Por Acuerdo 46/2006, de 29 de junio, el CoAN resolvió archivar las actuaciones seguidas contra Telelínea, por no quedar acreditada su responsabilidad en los hechos que se le imputaban en el referido procedimiento.

\subsubsection{Informe 2007}

Actuaciones del CoAN respecto al seguimiento de los contenidos emitidos por los operadores navarros de radio y televisión

La labor del CoAN no se agota en el seguimiento de las emisiones sino que tiene distintos tipos de respuesta ante los contenidos analizados. A continuación se describen las principales acciones del Consejo durante este año al respecto.

Expedientes sancionadores

En el ejercicio de sus funciones el CoAN tiene competencia sancionadora en el caso de que los operadores audiovisuales no cumplan con lo dispuesto en la normativa correspondiente. Durante el año 2007 se han tramitados los siguientes expedientes sancionadores.

Televisión 
El día 4 de junio de 2007 tuvo entrada en la Oficina de Defensa de la Audiencia una denuncia referida a los contenidos emitidos por el canal televisivo "Canal Latino" en la que se indicaba que, durante el fin de semana del 2 y 3 de junio, la reclamante presenció escenas de sexo en directo, no sólo en la programación nocturna sino también durante el día. En virtud del Acuerdo Plenario 51/2007, de 11 de junio, se ordenó la apertura de un periodo de información previa para el esclarecimiento de los hechos objeto de la denuncia, con el fin de conocer las circunstancias del caso concreto y la conveniencia o no de iniciar un procedimiento sancionador.

De los informes técnicos solicitados, tanto a la Subdirección General de Inspección y Supervisión del Ministerio de Industria, Turismo y Comercio como a la empresa pública Opnatel, se desprende que el titular de la emisora "Canal Latino" es la empresa Infraestructuras y Gestión S.L., que se trata de una emisión de televisión analógica terrenal y que se recibe en la comarca de Pamplona. Consultado el Registro de Titularidad de Medios de Comunicación Audiovisual se comprueba que no existe ninguna concesión de televisión local a favor de la empresa Infraestructuras y Gestión S.L.

Por Acuerdo 2/2008, de 10 de enero, del CoAN se acordó dar traslado de todo lo actuado al Departamento de Relaciones Institucionales y Portavoz del Gobierno, a los efectos que fueran oportunos, al ser el órgano competente para ejercer la labor de inspección, control y sanción de las emisoras que operan sin título habilitante.

\subsection{Consejo Audiovisual de la Comunidad de Madrid}

(Noticia publicada por la Comunidad de Madrid el 31 de marzo de 2005)

Impone una multa de 1,5 millones a los vecinos de la Torre de Valencia por la instalación de antenas en la azotea. 
La Comunidad ordena el cierre 21 televisiones locales y 33 emisoras de radio ilegales de la región.

El Gobierno anuncia un programa de inspecciones para clausurar todas las emisoras sin licencia.

Si las empresas no cierran voluntariamente, el Gobierno procederá al precinto con incautación de los equipos.

La medida forma parte del paquete de medidas primer Plan Audiovisual de la Comunidad de Madrid

La Comunidad de Madrid ha iniciado las acciones administrativas y judiciales para el cierre de las emisiones de 21 televisiones locales y de 33 emisoras de FM que se mantienen operativas en la región sin disponer de la pertinente concesión administrativa. Esta medida, que ha conocido hoy el Consejo de Gobierno, forma parte del paquete de medidas del primer Plan Integral del Sector Audiovisual de la Comunidad de Madrid y se hará extensiva paulatinamente a todas las emisoras que emiten ilegalmente su programación en la Comunidad de Madrid, según informó hoy el vicepresidente del Gobierno regional, Ignacio González.

Asimismo, el Gobierno regional ha impuesto una multa por valor de un millón y medio de euros a la Comunidad de Vecinos del inmueble conocido como la Torre de Valencia, situado en la calle de O’Donnell, número 4. Este edificio se había convertido en el principal centro de emisiones ilegales de la región ya que los vecinos permitieron la instalación de antenas en su azotea gracias a las cuales emitían un total de 14 televisiones locales y cuatro radios de FM. Los vecinos ya han recibido la notificación de la sanción por esta práctica ilegal. 
El cierre de las televisiones y radios anunciado hoy ha sido objeto de dialogo con el sector audiovisual de la Comunidad de Madrid; en especial, se han mantenido reuniones con la Asociación de Televisiones Locales de la Comunidad de Madrid, la Asociación Española de Radiodifusión Comercial, y con el Ministerio de Industria, Turismo y Comercio.

No se han presentado al concurso para obtener las licencias

La Comunidad de Madrid ya ha procedido al requerimiento de cierre de un total de 21 televisiones locales, 12 de las cuales se ubican en la capital.

Estas televisiones locales actualmente emiten en la región y no se han presentado al concurso público de televisión digital local. En caso de que el cierre no fuera voluntario por parte de los dueños de las cadenas, el Gobierno regional procederá a la resolución de cierre y recurrirá a la vía judicial, con incautación de los equipos de $\mathrm{TV}$, en virtud de la facultad prevista en el artículo 26 de la Ley de Televisiones Privadas.

A estas 21 televisiones locales se sumarán aquellas otras que actualmente emiten y que no obtengan una concesión de televisión digital, cifra que ascenderá a 16. Al concurso público se han presentado un total de 115 ofertas y 44 empresas distintas, y será resuelto en las próximas semanas. En virtud de este concurso, podrán emitir en la Comunidad un total de 53 televisiones, lo que fomentará la producción audiovisual regional, que ya supone el 75\% de la producción audiovisual nacional. Las 21 TV Locales cuyo cierre se va a instar son las siguientes:

Canal 33

Telesur Madrid

Canal 53 
TLSUR Madrid

Tele Cuatro Caminos

Medios Audiovisuales

Citae

Televisión Amistad

Pozuelo TV

Canal Televisión COS

EWTN

Telemadroño

Madrid Local

Madrid Sur TV

Arganda TV

Telelocal

Telelínea

Infraestructuras y Gestión

Telenoroeste

Popular TV Navalcarnero

Telecomunicaciones Rodríguez

S.A.R.

Evitar abusos y estafas

Estas actuaciones constituyen la primera fase del Programa de Inspecciones de la Comunidad de Madrid que va a desarrollar la Secretaría General del Consejo de Gobierno, como órgano competente en esta materia. De este modo, se da comienzo a la ejecución de una de las medidas previstas en el primer Plan Integral del Sector Audiovisual de la Comunidad de Madrid, que fue presentado en la Real Casa de Correos el pasado 22 de febrero. 
Con estas medidas, la Comunidad de Madrid pretende ordenar el espacio radioeléctrico en beneficio, en primer lugar, de todos los ciudadanos de la Región, por cuanto se quiere garantizar unas emisiones de televisión y de radio de calidad, así como evitar los abusos y estafas que en algunos casos se producen a los consumidores (como ha ocurrido con Telesierra); y en segundo lugar, supone un apoyo explicito al sector audiovisual de la Comunidad de Madrid al asegurarse que las radios y televisiones con concesión administrativa no se vean afectadas por emisiones piratas, con los perjuicios económicos y de pérdida de empleo que ello supone.

Sanción a los vecinos de la Torre de Valencia

Del mismo modo que la Comunidad de Madrid ha sancionado a la comunidad de propietarios de la Torre de Valencia por las infracciones de estalaciones radioeléctricas sin autorización, y obstrucción a la actuación inspectora de la Comunidad de Madrid, el Gobierno regional actuará contra los propietarios de los inmuebles de nuestra Región donde se ubiquen emisoras ilegales, con el fin de luchar contra los abusos y fraudes que éstas cometen.

\subsection{Comunidad Autónoma de Aragón}

Según nuestros informes, las televisiones que operan sin licencia en Aragón son: La Villa, La Torre de Babel, Boggi, EB 88 y Canal 44.Este último está denunciado hace ya un año por ejercer su actividad sin licencia, ya que tiene la licencia de la DGA pero no tiene la de obra, ni la puede tener ya que está construida sobre un suelo protegido, con lo cual carece de licencia de actividad. Emite pornografía a partir de las 00:00, cosa que es legal. Los otros a todas horas. 


\subsection{Comunidad Autónoma de Murcia}

El 6 de Mayo de 2008, un informe de la Asociación de Radio y Televisión detectó que en la Región existían 8 televisiones "alegales" y 40 sin licencia

Informó en rueda de prensa su presidente, José García Solano, quién dijo que hay más emisoras ilegales que en Madrid

Las emisoras de televisión sin autorización administrativa y que son alegales en virtud de la disposición transitoria primera de la ley 41/1995 de televisión local, emiten con anterioridad al 1 de enero de 1995 RTV Peñón; Canal 21; Televisión Molina; Canal 6 Totana; TVM; Onda 7 TV; A.D.C. El Pinacho, y Tele Águilas.

Entre las televisiones sin licencia administrativa se encuentran TV Murciana; Onda 7; Canal 6; Localia; Canal 21; Televisión de Lorca; Info TV; Canal Murcia; Vía Local, Canal Latino; Tele Águilas; Canal 40; Vit Chanel; Popular TV y Telelinea.

\subsection{Comunidad Autonómica Valenciana}

Se han iniciado cinco expedientes sancionadores por la Generalitat, desde finales del año pasado, contra televisiones terrestres analógicas locales que emiten sin concesión administrativa, con contenidos de videncia, tarot, televenta, chats de contactos y teléfonos eróticos. Según hemos sabido existe la voluntad de seguir detectando estas emisiones ilegales de televisión e incoando los correspondientes expedientes sancionadores. 


\section{Enmienda 177 que el Gobierno Socialista quiso plantear ante el Parlamento pero que finalmente retiró}

La ley de la Información permitirá el cierre automático de radios y televisiones sin licencia.

Una enmienda socialista al texto legal preveía la suspensión de las empresas infractoras antes de que llegue el apagón analógico de 2010. La enmienda 177 establecía el cierre de las emisoras de radio y TV como medida cautelar: "La prestación de un servicio de radio o televisión sin título habilitante, con independencia del tipo de servicio de comunicaciones electrónicas utilizado para su transmisión, se tipifica como infracción muy grave y dará lugar a la aplicación del oportuno régimen sancionador, pudiendo adoptarse como medida de carácter provisional el cierre de la actividad. Esta infracción implicará una multa económica de entre 60.000 y 1.000 .000 euros".

Miles de empresas de radiodifusión y televisión en España podrían tener las horas contadas. El Gobierno de Rodríguez Zapatero quiere ver aprobada antes del final de la presente legislatura, una ley de lo audiovisual en el que la autoridad gubernativa tendrá facultades para cerrar por decreto y de forma automática aquellas emisoras de radio de FM o canales de televisión local que se encuentren emitiendo sin la preceptiva licencia.

Hasta la fecha, la actividad de estas empresas se justificaba por cierta situación de alegalidad, aprovechando esquinas legales poco o nada contempladas. Se suponía que tales actividades iban a tener fecha de caducidad, el apagón analógico previsto para 2010, pero con la enmienda no habría hecho falta llegar tan allá para echar el cerrojo. 
Tal cerrojazo se intentó a través de una enmienda presentada por el propio Grupo parlamentario Socialista en el Congreso de los Diputados. La enmienda señalaba que «para la prestación de cualquier servicio de difusión de radio y televisión será preceptiva la previa obtención del correspondiente título habilitante que se regirá por la normativa reguladora de cada servicio».

Con la futura ley en la mano, los cierres pueden ser automáticos. Un texto legal que califica como «falta muy grave» emitir sin licencia. Parece lógico que así sea, pero la realidad es que hay en España miles de empresas, en su mayoría de pequeño calibre económico y de difusión, que se encuentran en esta situación alegal hoy, pero ilegal dentro de poco tiempo.

Las faltas muy graves las castigará la ley de lo audivisual, si no hay cambios de última hora, con multas entre 60.000 y un millón de euros, que se calcularán según distintos baremos, el principal de los cuales es el beneficio que haya sacado el infractor con su cadena o emisora. También se valorará el grado de cobertura de la empresa infractora. Todo esto se habría establecido hace un año si el PSOE hubiera presentado su enmienda. Ahora debemos esperar a que salga la nueva Ley de lo Audiovisual.

\subsection{Cortes Generales. Diario de sesiones del Congreso de los Diputados.}

\section{Comisiones}

Esto es lo que pasó en el Congreso de los Diputados el día que el PSOE iba a presentar su enmienda:

Año 2007 - VIII Legislatura - Núm. 929

INDUSTRIA, TURISMO Y COMERCIO

PRESIDENCIA DEL EXCMO. SR. D. ANTONIO CUEVAS DELGADO 
Sesión núm. 57 celebrada el martes, 23 de octubre de 2007

\section{ORDEN DEL DÍA:}

Ratificación de la ponencia designada para informar el proyecto de Ley de medidas de impulso de la sociedad de la información. (Número de expediente 121/000134)

Emitir dictamen, a la vista del informe elaborado por la ponencia, sobre el proyecto de Ley de medidas de impulso de la sociedad de la información. (Número de expediente 121/000134.)

El señor PRESIDENTE: Tiene la palabra el señor Echániz, del Grupo Parlamentario Popular.

El señor ECHÁNIZ SALGADO: [...]La segunda enmienda es la relativa a los centros radiofónicos y televisivos de carácter analógico - nos parece todavía más grave que la anterior -, por la cual el Gobierno pretende cerrar cualquier emisora de radio o de televisión que emita sin licencia o sin título habilitante en esta situación de alegalidad; además quiere imponer a sus propietarios una sanción de hasta un millón de euros, lo que, dado el actual mapa de emisoras de frecuencia modulada y de televisión local, muchas de las cuales están todavía en una situación de alegalidad, repito, si se aprueba la enmienda y se adoptan estas medidas, puede suponer un auténtico terremoto en el panorama audiovisual. Lo curioso de esta medida tan drástica, señorías, es que se introduzca también de tapadillo, a final de la legislatura y cuando ya nos encontramos de facto en la precampaña electoral, por lo que ya no solo es legítimo sino casi obligado preguntarse contra quién va esta medida y a quién va a cerrar las emisoras de radio y de televisión el Gobierno. [...]

El señor PRESIDENTE: Por último, por el Grupo Parlamentario Socialista tiene la palabra la señora Muñoz. 
La señora MUÑOZ SANTAMARÍA: [...] Respecto a las enmiendas del Grupo Socialista, quiero anunciar, aunque ya se lo hemos comunicado a muchos grupos evidentemente, la retirada de las enmiendas números 176, 177 y 178, referidas tanto a la regulación de la radio por satélite o televisión en movilidad como al régimen sancionador de las televisiones sin título habilitante. Nosotros hemos preferido, una vez hecho el debate, dejar todos estos temas referidos a la próxima ley de lo audiovisual, que tiene que hacerse, porque creemos que es mucho más coherente y que se ha de abordar de una manera global. No hay mala intención y creo que han sobrado algunos calificativos que ha realizado el Grupo Popular, como el de cacicada. Ni mucho menos, no hay ninguna cuestión oculta en estas enmiendas; simplemente hay una intención de poder regular un tipo de televisión o agilizar la acción del Gobierno con algunas televisiones que emiten ilegalmente. Después de hablar con todos los grupos, hemos creído que era mejor retirarlas en este momento e incluirlas cuando se haga la ley de lo audiovisual.

\section{Conclusión}

En la actualidad, la situación del sector es confusa. Los operadores que están emitiendo se dividen entre los "alegales" o los directamente ilegales, (según los expertos se llama alegal a aquello que se consiente pero que no posee los requisitos que se establecen para ser legales).

La ley de 1995 de Televisión Local por Ondas Terrestres consagró las televisiones locales que operaban en ese momento y prohibió explícitamente la aparición de nuevas. Pese a todo, el sector ha seguido creciendo desde entonces sin ningún tipo de control. En estos momentos existen centenares de televisiones locales emitiendo de forma "alegal" por existir un vacío administrativo motivado por el miedo a desarrollar de una vez por todas la ley de televisión. 
El partido socialista tenía verdadero terror a estas televisiones, y los populares tienen en el cajón una ley que no se atreven a presentar en primer lugar por?que su formato no es válido para todo el territorio español, y en segundo lugar porque obligará a legalizar a las que ya están emitiendo desde hace años.

Fuentes del Ministerio de Industria señalan que uno de los objetivos que persigue la futura ley es atajar este desorden, de manera que se otorgue cobertura legal a los operadores locales. Después, vendrá una tarea de lucha contra las emisoras ilegales, que podrán ser sancionadas con multas de entre 60.000 y un millón de euros.

Sin embargo, quizá la incógnita es porqué la Ley de lo Audiovisual, o alguna otra enmienda o ley que penalice a las televisiones sin título habilitante, no se ha dado todavía en España. Máxime cuando la directiva de la CEE de 3 de octubre de 1989 sobre la coordinación de determinadas disposiciones legales, reglamentarias y administrativas de los Estados miembros relativas al ejercicio de actividades de radiodifusión televisiva, ya lo dejaba claro en su Artículo 2: "Cada Estado miembro velará porque todas las emisiones de radio difusión televisiva transmitidas por organismos de radiodifusión televisiva bajo su jurisdicción respeten las normas de Derecho aplicables a las emisiones destinadas al público en ese Estado miembro".

La explicación más plausible de que no se haya establecido todavía una "caza de brujas" es que se espera que se puedan eliminar las televisiones sin licencia con el apagón analógico de final de este año, ya que el estado y las comunidades autonómicas tendrán el poder de regular la entrada en la TDT de aquellas cadenas que no cumplan todos los requisitos legales.

Visto que las autoridades no consiguen atajar esta situación, y sabiendo el dinero que genera este negocio, más de uno se preguntará si esto quedará impune o si se les 
aplicará una multa con carácter retroactivo a los responsables. Se nos responderá con la nueva ley de lo audiovisual. ¿Cuándo? La pelota está en el tejado de los políticos. 